\title{
Do projeto ao manual do proprietário: aplicações em Realidade Aumentada
} From the project to the Owner's Manual: applications in Augmented Reality

\author{
Dênio Tarcísio Torquato Cerqueira Batista \\ Universidade Federal da Bahia | Salvador | Brasil | denio.tarcisio@ufba.br
}

Paula Pontes Mota

SIPPRO Engenharia | Fortaleza | Brasil | paula.mota@sippro.eng.br

Lorena Claudia de Souza Moreira

Universidade Federal da Bahia | Salvador | Brasil | lorenasm@ufba.br

\section{Resumo}

As tecnologias digitais atuam como agentes transformadores da percepção da realidade nos dias atuais e, neste contexto, é notável a capacidade da tecnologia da realidade aumentada (RA) de atuar na potencialização do entendimento de atividades desenvolvidas no campo da Arquitetura, Engenharia, Construção e Operação (AECO). Desde a fase de projeto até a fase de operação e manutenção a RA atua aprimorando tarefas e mitigando esforços. Sendo assim, este artigo, resultante de um projeto de Iniciação Científica, tem como objetivo explicitar implementações de RA nas áreas de arquitetura e operação, seus benefícios e limitações. $A$ pesquisa adotou o método Design Science Research e a partir de modelos BIM, por meio de experimentos práticos, os resultados explicitaram avanços na visualização do Projeto ao Manual do Proprietário.

Palavras-chave: Realidade aumentada. Projeto. Operação e manutenção. Building Information Modeling.

\begin{abstract}
Digital technologies act as transforming agents of the reality perception today and, in this context, the capacity of augmented reality (AR) technology to act in enhancing the understanding of activities developed in the field of Architecture, Engineering, Construction, and Operation (AECO) is remarkable. From the design phase to the operation and maintenance phase, AR works to improve tasks and mitigate efforts. Therefore, this paper, resulting from a Scientific Initiation project, aims to explain AR implementations in the areas of architecture and operation, their benefits, and limitations. The research adopted the Design Science Research method. Based on BIM models and through practical experiments, the results showed advances in the project visualization to the building owner's Manual.
\end{abstract}

Keywords: Augmented reality. Design. Operation and maintenance. Building Information Modeling.

\section{INTRODUÇÃO}

A maneira que se percebe a realidade está em constante transformação e a tecnologia digital contribui para as novas formas de visão de mundo. A mutação dos dispositivos informacionais cria novas maneiras de pensar e conviver [1] e a tecnologia da realidade

Como citar:

BATISTA, D. T. T. C.; MOTA, P. P. .; MOREIRA, L. C. de S. . Do projeto ao manual do proprietário: aplicações em realidade aumentada. In: SIMPÓsIO BRASILEIRO DE TECNOLOGIA DA INFORMAÇÃO E COMUNICAÇÃO NA CONSTRUÇÃO, 3., 2021, Uberlândia. Anais [...]. Porto Alegre: ANTAC, 2021. p. 1-10. Disponível em: https://eventos.antac.org.br/index.php/sbtic/article/view/630. Acesso em: 3 ago. 2021. 
mista, em que a realidade aumentada (RA) está inserida, atua como um potencial agente transformador dessa nova realidade.

A realidade aumentada é uma tecnologia que permite transportar o ambiente virtual para o seu espaço em tempo real, utilizando um dispositivo tecnológico, podendo usar a interface do ambiente real para manusear objetos reais e virtuais. Sendo assim, a realidade aumentada em linhas gerais é a inserção de elementos virtuais no mundo real [2].

Neste âmbito, no universo da Arquitetura, Engenharia, Construção e Operação (AECO) observa-se, ainda em fase de projeto, o desenvolvimento de pesquisas visando o auxílio à visualização de soluções do projeto de arquitetura [3] e detecção de erros de projetos durante a montagem de estruturas de aço, por meio da RA [4], assim como estudos relacionados à visualização de componentes hidrossanitários objetivando identificar a localização dos mesmos quando inseridos na alvenaria [5].

Já na fase de operação e manutenção, a associação do modelo BIM com a RA para a visualização de dados, surge como um potencial campo de pesquisa para esta temática [6]. Essa associação pode ser feita através do Manual de uso, operação e manutenção das edificações, também conhecido como Manual do Proprietário (MP). Para [7] o MP é o documento que abarca todas as informações relacionadas a garantia e a manutenção dos elementos que constituem a edificação e delimita direitos e deveres do proprietário do imóvel.

No limiar da contemporaneidade, é cada vez mais necessária a utilização consciente e proveitosa dos recursos naturais, e tratando-se de uma edificação, uma atenção maior deve ser dada a esse quesito visto que o mesmo se utiliza de grande movimentação de recurso e altera significativamente o ambiente em que é posto. Sendo assim, é importante garantir uma maior eficiência possível de uma construção, bem como a manutenção do mesmo depois de entregue, para que sua vida útil seja utilizada de forma eficiente pela sociedade. Para isso, a RA se mostra uma tecnologia capaz de auxiliar na resolução dessas demandas propostas.

Diante do exposto, o presente artigo apresenta resultados de um projeto de iniciação científica da Faculdade de Arquitetura da Universidade Federal da Bahia desenvolvido no Laboratório de estudos avançados em Cidade, Arquitetura e Tecnologias Digitais (LCAD), cujo tema originou-se em tese de doutorado [8]. A pesquisa buscou inovar o Manual do Proprietário da Edificação por meio da Realidade Aumentada. Como objetivo desta pesquisa, por meio de duas plataformas de desenvolvimento, tem-se a explicitação dos benefícios e das limitações de visualizações em realidade aumentada nas áreas de arquitetura (projeto executivo) e operação (manual do proprietário).

\section{METODOLOGIA}

O método de pesquisa adotado foi o Design Science Research (DSR) que, em linhas gerais, busca resolver problemas práticos além de produzir conhecimento através da produção de artefatos. A pesquisa foi dividida em duas fases. A primeira fase envolveu a aquisição de conhecimentos por meio de uma Revisão Sistemática de Literatura (RSL) 
e a fase seguinte, de experimentos práticos. No presente artigo serão explicitados os experimentos práticos realizados na pesquisa que foram divididos em dois tipos de abordagem: (i) visualização do projeto, (ii) visualização do manual do proprietário. Para cada abordagem foram utilizadas dois tipos de plataformas: um aplicativo para RA e um motor de jogo (Game Engine). $O$ delineamento do processo será apresentado abaixo (Figura 1).

Figura 1: Delineamento do processo

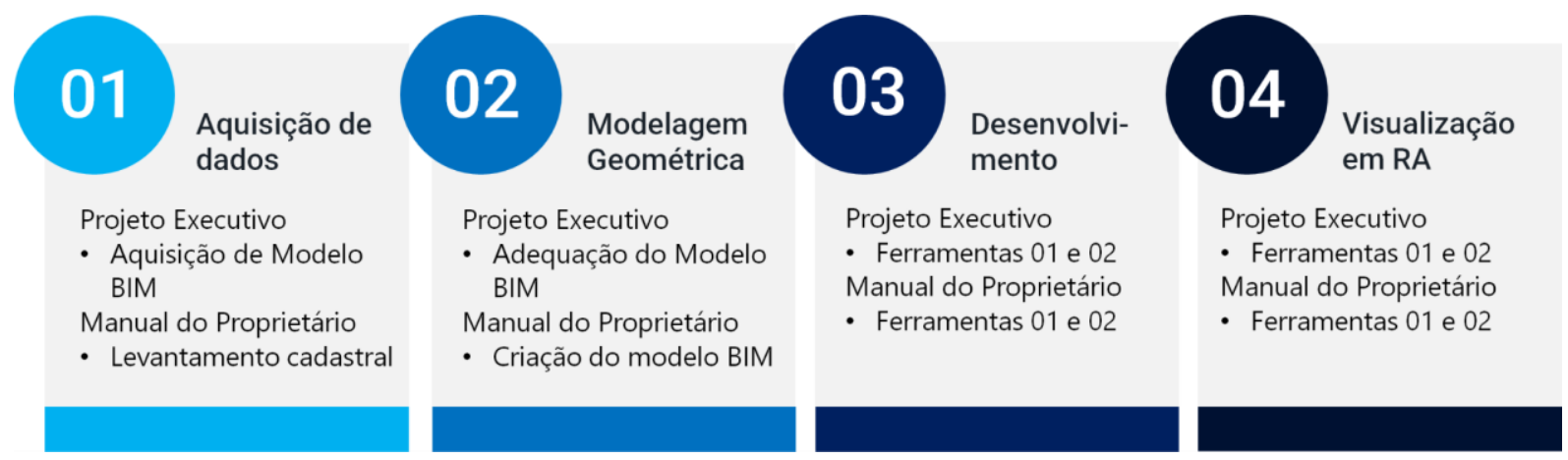

Fonte: os autores.

$\mathrm{Na}$ fase de aquisição de dados (01), para a visualização do projeto foi adquirido o modelo BIM de um projeto executivo de um edifício residencial, por meio de uma empresa parceira. Por sua vez, para a visualização do manual do proprietário foi realizado o levantamento cadastral de componentes de uma edificação. Subsequentemente, na fase de modelagem geométrica (02) para o projeto, foi realizada a adequação do modelo BIM adquirido, e para o MP foram modelados componentes hidrossanitários em ferramenta BIM. Já na fase de desenvolvimento (03), os modelos BIM foram transferidos para as duas plataformas de visualização em RA e, por fim, na fase de visualização em RA (04), o projeto executivo e o manual do proprietário foram vistos em RA nas plataformas. Todas essas etapas serão descritas na seção de implementação e resultados.

O dispositivo utilizado para os experimentos foi um Smartphone Motorola One Vision, com sistema operacional Android 9.0 e memória interna de 128GB com 4GB de RAM.

\section{IMPLEMENTAÇÃO E RESULTADOS}

Conforme explicitado no método, os experimentos foram divididos em dois tipos de abordagem: (i) visualização do projeto executivo e (ii) visualização do manual do proprietário. No experimento (i), foram escolhidos o pavimento tipo e as instalações hidrossanitárias do projeto executivo de um edifício cedido pela empresa SIPPRO. Por sua vez, o experimento (ii), foi realizado na área de serviço de um apartamento residencial. A seguir serão explicitadas as experimentações seguindo o delineamento proposto.

\section{PROJETO EXECUTIVO}

Na fase de aquisição dos dados (01), a empresa SIPPRO Engenharia disponibilizou os modelos BIM de arquitetura e instalações hidrossanitárias em fase de projeto 
executivo, juntamente com as pranchas técnicas e especificações de um empreendimento de uso residencial. O empreendimento em questão é caracterizado por possuir três torres, de 27 pavimentos cada, e três pavimentos de uso compartilhado, com equipamentos de lazer e espaço para circulação de veículos. 0 pavimento tipo utilizado no experimento possui área de aproximadamente $500 \mathrm{~m} 2$, sendo distribuída por quatro apartamentos e circulação vertical. Os dados escolhidos para o experimento restringiram-se ao pavimento tipo de arquitetura e das instalações hidrossanitárias em fase de projeto executivo.

$\mathrm{Na}$ fase de modelagem geométrica (02), os modelos utilizados foram desenvolvidos na ferramenta de autoria BIM Autodesk Revit 2019. Para realização dos experimentos, adequações precisaram ser feitas nos modelos recebidos visando o uso do modelo para Realidade Aumentada. Essas adequações envolveram a exclusão de elementos como forros, componentes elétricos e elementos textuais.

$\mathrm{Na}$ fase de desenvolvimento (03), foram realizados dois experimentos envolvendo as plataformas Augin (https://augin.app/) versão 3.10 e as Engines Unity (https://unity.com/pt) versão 2019.4.15f1 e Vuforia (https://developer.vuforia.com/) (versão 9.1).

No experimento com a plataforma Augin, após a exclusão de elementos desnecessários à visualização, foi elaborada uma área de visualização dos conteúdos a serem exibidos em RA. A partir dessa etapa, a plataforma gerou uma visualização na nuvem, que posteriormente disponibilizou esses elementos no perfil do usuário, previamente cadastrado.

Por sua vez, para a plataforma de desenvolvimento Unity, a partir do acesso ao ambiente de criação, foi realizada a inserção do modelo (formato FBX) exportado da ferramenta BIM de autoria e inserida a câmera para visualização em RA. O passo seguinte foi definir um marcador. Nesse caso, visando o enquadramento preciso do modelo com a mesma escala do desenho, a planta baixa técnica foi adotada como marcador. Uma vez definido, incluído e verificado, foi possível rastrear o marcador e observar o modelo no dispositivo, dando início à fase de visualização.

Na fase de visualização em RA (04), para o Augin, o rastreamento do marcador foi realizado em uma superfície plana para facilitar a inserção dos elementos virtuais. No momento da visualização, verificou-se que as texturas adotadas na fase de modelagem foram preservadas, além da possibilidade de visualização das informações técnicas dos elementos por meio de uma função disponível na plataforma. Quanto ao enquadramento, observou-se que os modelos não foram alocados de forma automática, necessitando de um posicionamento manual, realizado pelo usuário (Figura 2). Além disso, a plataforma dispõe de filtros de visualização que aplicam uma transparência ao modelo (Raio-x), e do modelo planificado.

Figura 2: Visualização em RA no Augin do Projeto Arquitetônico (à esquerda); Visualização em RA do Projeto de Instalações hidrossanitárias (à direita). 

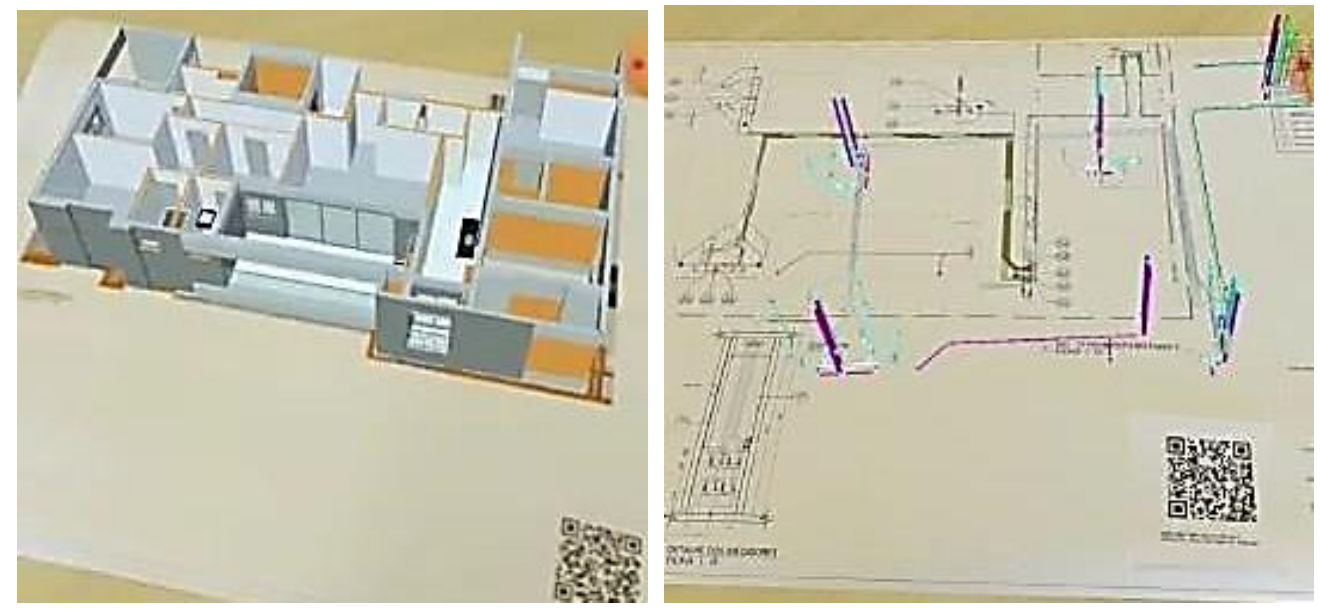

Fonte: os autores.

Para o Unity, a visualização em RA permitiu a troca ágil do modelo a partir das plantas baixas técnicas definidas como marcadores. Nesse caso, os modelos foram inseridos em um mesmo cenário e, com a alternância das plantas baixas impressas, os mesmos intercalaram-se de forma rápida, apresentando estabilidade e precisão no enquadramento. Essa característica se mostrou como um benefício por ter apresentado uma precisão do marcador para com os objetos em RA (Figura 3), além de ter possibilitado o acompanhamento da visualização por meio da movimentação da prancha impressa. Ademais, é importante destacar que, ao inserir os modelos no ambiente de criação do Unity, as texturas dos mesmos foram perdidas e a reinserção das mesmas está em desenvolvimento, bem como a fase de exibição das informações técnicas dos elementos.

Figura 3: Visualização em RA no Unity do Projeto Arquitetônico (à esquerda); Visualização em RA do Projeto de Instalações hidráulicas (à direita).

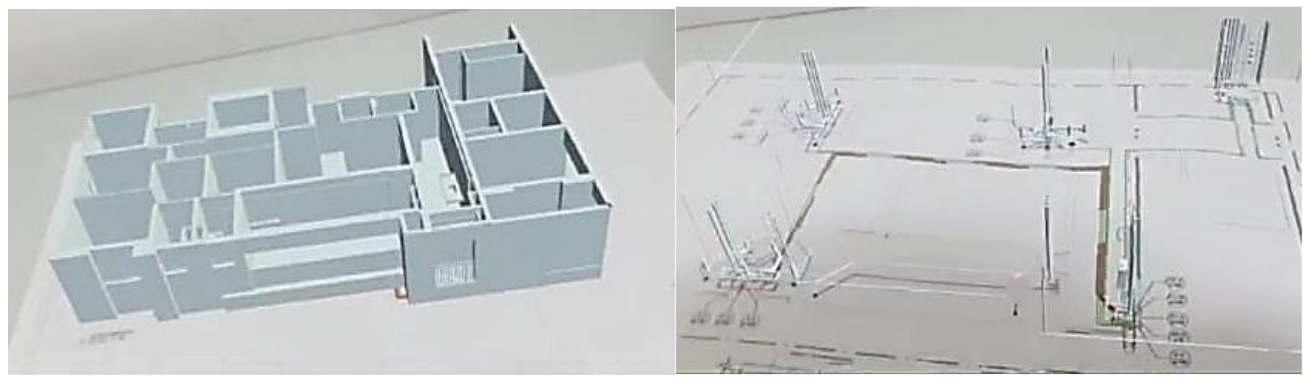

Fonte: os autores.

Apesar da ausência de textura no Unity e de enquadramento automático no Augin, em ambas plataformas se atingiu o objetivo esperado na visualização projetual. Dessa forma, independentemente da plataforma utilizada, a forma de visualização na fase de projeto, trouxe uma maior aproximação com a realidade e antecipação de resultados. Como benefícios da visualização em RA, apresenta-se a facilidade de esclarecimento de dúvidas e mitigação de erros projetuais, que por sua vez impactam em uma execução otimizada. Ademais, nessa fase, a RA é uma importante aliada na compatibilização e identificação de interferências de elementos. Identifica-se, como público principal para este tipo de visualização, a equipe de projeto e profissionais da construção civil. 


\section{MANUAL DO PROPRIETÁRIO}

$\mathrm{Na}$ fase de aquisição de dados (01), foi definido como ambiente para o experimento, a área de serviço de um apartamento de uso residencial, com área igual a $123 \mathrm{~m} 2$. Os dados foram adquiridos por meio de um levantamento cadastral, pela medição direta dos elementos da área em estudo, como bancada, louças, metais e elemento de apoio.

Para a fase de modelagem geométrica (02), utilizou-se os dados adquiridos no levantamento cadastral como parâmetros para a modelagem dos elementos. 0 modelo foi desenvolvido na ferramenta de autoria BIM, Autodesk Revit 2019. A modelagem da bancada e elemento de apoio foi realizada com opções nativas da ferramenta. Já os elementos correspondentes às peças hidrossanitárias, como cuba e torneiras, foram adquiridos diretamente dos respectivos fabricantes. $O$ uso dos elementos dos fabricantes permitiu que as informações extraídas fossem precisas, considerando modelo, especificações, dimensões e garantias.

Para auxiliar nas dimensões de distanciamento e locação do modelo geométrico em escala real, foi criado um volume genérico retangular na extremidade esquerda da bancada da pia, sendo essencial para o posicionamento correto dos elementos modelados, conforme apresentado na Figura 4.

Figura 4: Modelo geométrico desenvolvido em ferramenta BIM.

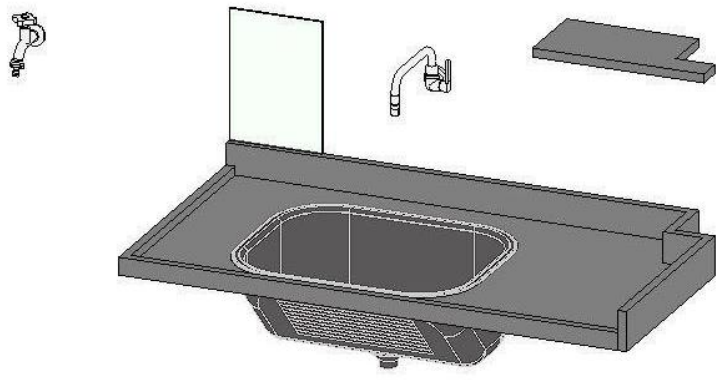

Fonte: os autores.

Por fim, ainda na fase de modelagem geométrica, houve uma correspondência das informações técnicas dos fabricantes com os parâmetros lidos pelas plataformas de visualização. Ou seja, foi necessário uma edição e manipulação dos dados dos elementos para garantir que as informações pudessem ser visualizadas em RA.

$\mathrm{Na}$ fase de desenvolvimento (03), as mesmas plataformas mencionadas anteriormente foram utilizadas nos experimentos envolvendo o manual do proprietário: Augin e Unity.

Para o Augin, foram realizados os mesmos procedimentos de upload do modelo do experimento anterior. O passo seguinte foi a utilização da opção "Reference Tracker", disponibilizada pela plataforma, para obtenção do alinhamento dos elementos com o auxílio da geometria criada anteriormente. Esse alinhamento foi feito no ambiente do Augin (https://my.augin.app/), a partir da inserção de um marcador virtual em uma superfície do modelo e do posicionamento de um marcador real, como apresentado na Figura 5. 
Figura 5: Posicionamento de marcador na plataforma Augin
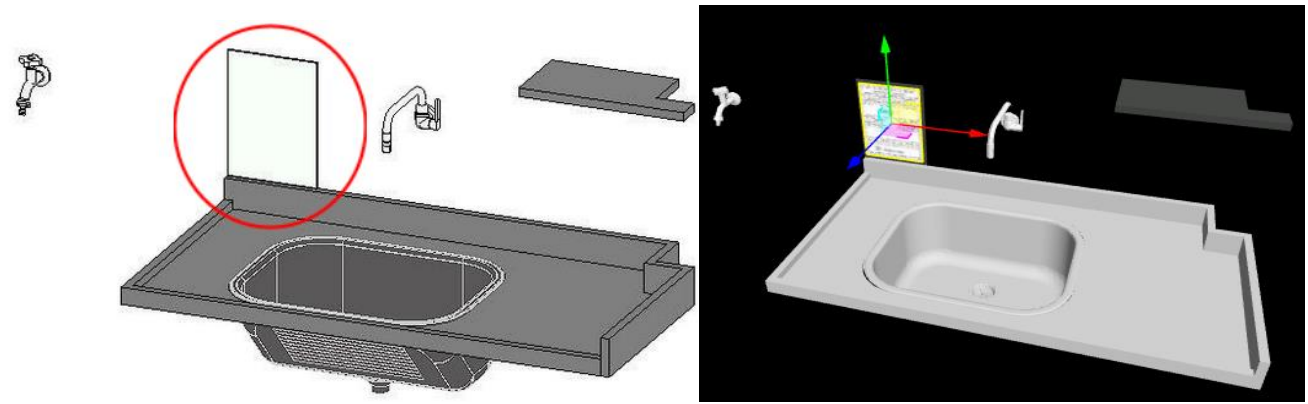

Fonte: os autores.

Para o experimento no Unity, foram utilizados os mesmos procedimentos iniciais realizados anteriormente, da habilitação da engine à inserção do modelo geométrico no ambiente de criação. Em seguida, foi preciso fazer o upload de uma imagem para gerar um marcador no site do Vuforia. Para isso, foi utilizada uma imagem retangular genérica, importada para o site com dimensões iguais às do marcador impresso. Assim, o marcador foi fixado em relação ao modelo no mesmo lugar em que o "Reference Tracker" foi posicionado no experimento com a plataforma Augin, para que fosse possível obter uma comparação entre as visualizações. Por fim, o projeto foi compilado no arquivo de formato APK e posteriormente foi transferido para o dispositivo Android, por meio de um aplicativo. Ao abri-lo, foi possível posicionar a câmera para rastrear o marcador físico e ter acesso a visualização.

Na fase de visualização em RA (04), durante a observação na plataforma Augin, o alinhamento entre o modelo e o objeto real foi obtido como demonstrado na Figura 6 (à esquerda). No entanto, a visualização oscilou e repetidamente foi necessário rastrear novamente o marcador para alinhar o modelo virtual com o objeto real. No filtro de visualização "Raio-X" da plataforma foi possível notar que, apesar da instabilidade, ocorreu um alinhamento satisfatório, como visto na Figura 6 (à direita).

Figura 6: Visualização em RA (à esquerda); filtro de visualização em “Raio-X” (à direita)
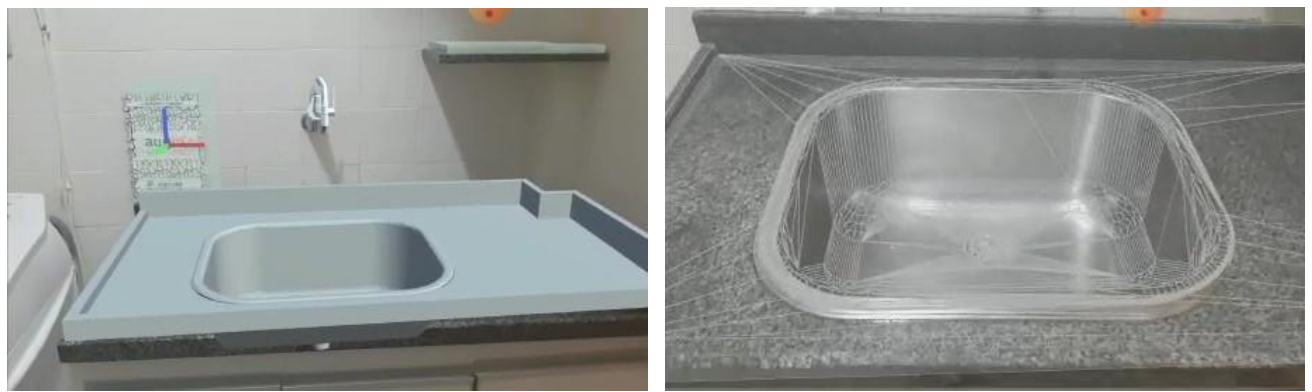

Fonte: os autores.

Ainda com a plataforma Augin, as informações de garantia dos componentes hidrossanitários contidos no manual do fabricante, que foram inseridas anteriormente, foram visualizadas na plataforma. Nos experimentos realizados, apesar das informações terem sido visualizadas, percebeu-se a necessidade de uma melhoria da apresentação das mesmas durante a inserção, pois os textos apresentados estavam sobrepostos ao modelo, como mostra a Figura 7. 
Figura 7: Tela de visualização das informações IFC

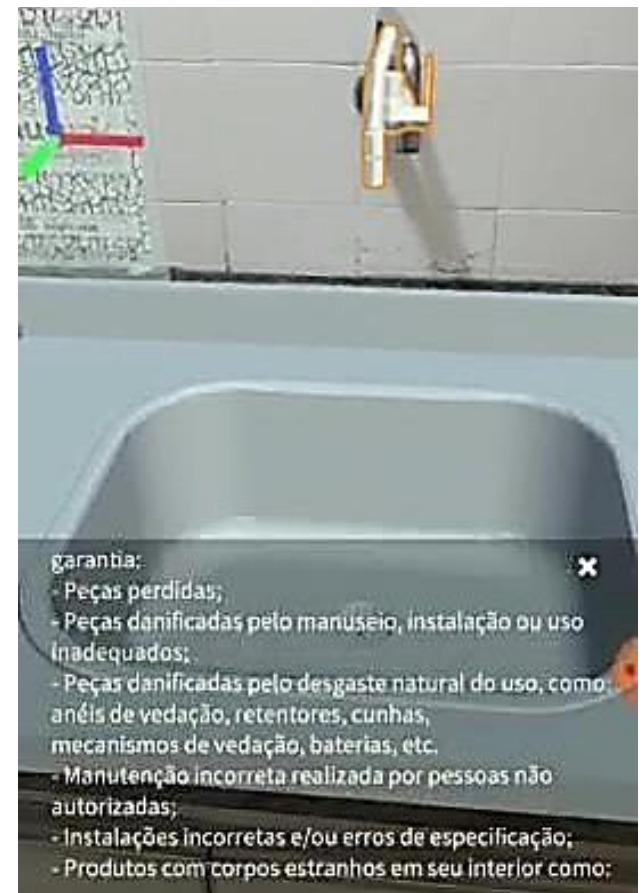

Fonte: os autores.

Já na plataforma Unity, inicialmente foi obtida a visualização em RA com certa instabilidade no enquadramento, como visto na Figura 8 (à esquerda). Com isso, houve a necessidade de repetir o processo de rastreamento do marcador para obtenção do enquadramento com o objeto real de forma satisfatória, como demonstrado na figura 8 (à direita). Contudo, apesar do problema do enquadramento ter sido solucionado, verificou-se: (i) que a visualização dos objetos virtuais oscilou da mesma forma que no aplicativo Augin; (ii) uma acentuação na instabilidade dos objetos virtuais quando há uma maior distância entre a câmera e o marcador; (iii) que a iluminação do ambiente real influenciou diretamente na qualidade da visualização e rastreamento do marcador. Ademais, é importante pontuar que a fase da inserção das informações técnicas dos elementos hidrossanitários, e demais funcionalidades, encontra-se em desenvolvimento.

Figura 8: Instabilidade da visualização (à esquerda); Enquadramento do modelo (à direita)
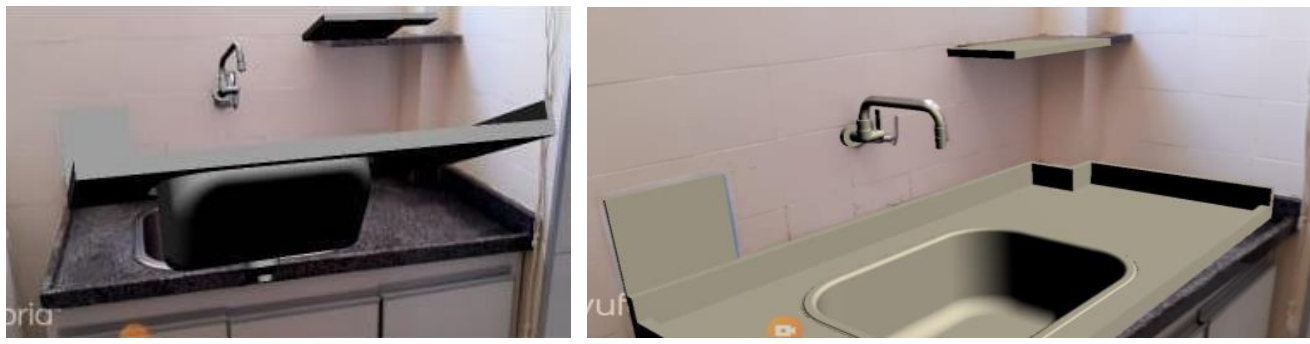

Fonte: os autores.

Mesmo com a instabilidade do modelo virtual em ambas as plataformas e limitações no ambiente real, para o manual do proprietário, esta forma de visualização, trouxe uma aproximação imediata com os elementos da edificação uma vez que estava na escala natural. Como principal benefício destaca-se a possibilidade do acesso às informações técnicas de fabricantes e garantias, bem como instruções de manutenção. 
Ademais, identificou-se o público principal para este tipo de visualização os gestores de condomínios e os usuários da habitação.

\section{CONSIDERAÇÕES FINAIS}

A partir do desenvolvimento do trabalho, foi possível demonstrar implementações de RA nas áreas de arquitetura e operação, seus benefícios e limitações. Neste sentido, no âmbito da visualização projetual e da operação e manutenção, as experimentações práticas apresentaram o potencial da tecnologia da RA, por meio de duas plataformas de desenvolvimento.

Diante das limitações observadas, destacam-se a instabilidade na visualização, a falta de reconhecimento das texturas em uma das plataformas e a necessidade de adequação das informações técnicas. Sobre a instabilidade verificada, vale ressaltar que a precisão da visualização quando estabilizada, é da ordem de centímetros.

Como benefícios, destacam-se a fácil inserção e manipulação dos modelos em ambas plataformas experimentadas, o reconhecimento inteligente do marcador aplicado na planta baixa e os resultados favoráveis no quesito de visualização.

Como conclusão, percebe-se a clareza da visualização em Realidade Aumentada como auxílio na compreensão dos experimentos que simulam a fase de projeto (aplicado ao projeto executivo), e a fase de operação e manutenção (aplicado ao manual do proprietário). Ademais, foram identificados potenciais agentes para cada área de atuação, sendo a equipe de projeto e execução para a fase de projeto, e os gestores de condomínios e os usuários da habitação para a fase de operação e manutenção.

\section{AGRADECIMENTOS}

Agradecimentos ao Programa Institucional de Bolsas de Iniciação em Desenvolvimento Tecnológico e Inovação (PIBITI), pela bolsa de pesquisa fornecida a um dos autores è empresa SIPPRO Engenharia, por ter cedido a modelagem de um dos experimentos realizados.

\section{REFERÊNCIAS}

[1] LÉVY, Pierre. As Tecnologias da Inteligência: o futuro do pensamento na era da informática. Rio de Janeiro: Editora 34, 1993.

[2] SILVA, Adriana Simões de Sales Dias e. Uso de Recurso Educacional com Mídias Interativas e Integradas On-Line em Ensino e Aprendizagem. 2013. 120 f. Dissertação (Mestrado Profissional em Ensino de Ciências) - Universidade Federal de Itajubá, Itajubá, 2013.

[3] MOREIRA, L. C. de S.; RUSCHEL, R. C. Realidade Aumentada na Visualização de Soluções do Projeto de Arquitetura, p. 192-196. In: CONGRESSO DA SOCIEDADE IBEROAMERICANA DE GRÁFICA DIGITAL. Anais [...]. São Paulo: Editora Edgard Blücher, 2015. p. 192-196. DOI 10.5151/despro-sigradi2015-50384.

[4] HARA H.; KOMATSU R.; SHIOTA, N. Architecture for Digital Technology Utilization to Accelerate Digital Innovation. Fujitsu Scientific \& Technical Journal, v. 54, n. 3, p. 39-46, 2018. 
[5] LIU, F.; SEIPEL, S. Precision study on augmented reality-based visual guidance for facility management tasks. Automation in Construction, [S.L.], v. 90, p. 79-90, jun. 2018. Elsevier BV. http://dx.doi.org/10.1016/j.autcon.2018.02.020.

[6] MOREIRA, Lorena; RUSCHEL, Regina. Realidade Aumentada para a montagem, manutenção e operação da edificação: revisão sistemática de literatura. In: SBTIC + SIBRAGEC, 10., 2017, Fortaleza. Anais [...]. Fortaleza, 2017. p. 186-195.

[7] MOREIRA, L.; TONOLI, J. G.; RUSCHEL, R. C. A prática do manual do proprietário da edificação: uma classificação conforme a NBR14037. Gestão \& Tecnologia de Projetos, v. 13, n. 3, p. 119-134, 2018.

[8] MOREIRA, Lorena Claudia de Souza. O manual do proprietário da edificação assistido pela Realidade Aumentada. 2018. 1 recurso online (241 p.) Tese (doutorado) Universidade Estadual de Campinas, Faculdade de Engenharia Civil, Arquitetura e Urbanismo e Missouri State University, Campinas, SP. Disponível em:

http://repositorio.unicamp.br/handle/REPOSIP/334148. Acesso em: 29 jun. 2021. 